\title{
Improvement of Bit Error Rate in Fiber Optic Communications
}

\author{
Jahangir Alam S. M., M. Rabiul Alam, Hu Guoqing, and Md. Zakirul Mehrab
}

\begin{abstract}
The bit error rate (BER) is the percentage of bits that have errors relative to the total number of bits received in a transmission. The different modulation techniques scheme is suggested for improvement of BER in fiber optic communications. The developed scheme has been tested on optical fiber systems operating with a non-return-to-zero (NRZ) format at transmission rates of up to $10 \mathrm{Gbps}$. Numerical simulations have shown a noticeable improvement of the system BER after optimization of the suggested processing operation on the detected electrical signals at central wavelengths in the region of $1310 \mathrm{~nm}$.
\end{abstract}

Index Terms-BER, modulation techniques, SNR, NRZ, improvement.

\section{INTRODUCTION}

Optical fibers are widely used in fiber optic communications which permits transmission over longer distances and at higher bandwidths than other forms of communication. Optical transmission networks based on wavelength division multiplexing (WDM) architecture is dominating the all-optical data transportation with bit rates exceeding several terabit-per-second rates to serve the ever increasing demand of Internet Protocol (IP) networks. Some of the main TCP/IP networking functions such as routing, add-drop multiplexing and demultiplexing and wavelength conversion, need to be functional to encapsulate the IP packet requirements into the Optical layer. The linear as well as the nonlinear characteristics of the optical fiber at higher bit rates, seriously limit the data transmission performance and it is therefore becoming necessary to develop approaches to improve regeneration of transmitted data. Experimental investigations have shown a considerable progress in this direction. These were based on compensation techniques, filtering, developing optimized line coding, and further dispensation of received signal. In a communication system, the receiver side BER may be affected by transmission channel noise, interference, distortion, bit synchronization problem, attenuation, wireless multipath fading, etc. The BER can be considered as an approximate estimate of the bit error probability which is the expectation value of the BER. The approximation is accurate for a long studied time interval and a high number of bit errors [1].

Manuscript received February 17, 2014; revised March 27, 2014.

Jahangir Alam S. M. and Hu Guoqing are with the Department of Mechanical and Electrical Engineering, Xiamen University, China (e-mail: jahangir_uits@yahoo.com,gqhu@xmu.edu.cn).

M. Rabiul Alam is with the Department of Electrical \& Electronic Engineering, Hamdard University Bangladesh, Bangladesh (e-mail: alam2007@ mail.ru).

Md. Zakirul Mehrab is with the Spark Bangladesh Ltd., Dkaka, Bangladesh (e-mail: jakirul2001@yahoo.com).

\section{BIT ERROR RATE AND SIGNAL-TO-NOISE RATIO}

In telecommunication transmission, the bit error rate (BER) is the percentage of bits that have errors relative to the total number of bits received in a transmission. For example, a transmission might have a BER of $10^{-6}$, meaning that, out of $1,000,000$ bits transmitted, one bit was in error. The BER is an indication of how often data has to be retransmitted because of an error [1]. Too high a BER may indicate that a slower data rate would actually improve overall transmission time for a given amount of transmitted data since the BER might be reduced, lowering the number of packets that had to be present. The BER may be improved by choosing a strong signal strength (unless this causes cross-talk and more bit errors), by choosing a slow and robust modulation scheme or line coding scheme, and by applying channel coding schemes such as redundant forward error correction codes [2]. The transmission BER is the number of detected bits that are incorrect before error correction, divided by the total number of transferred bits (including redundant error codes). Normally the transmission BER is larger than the information BER. The information BER is affected by the strength of the forward error correction code. Sinusoidal driven resonators having higher quality factors (Q) resonate with greater amplitudes (at the resonant frequency) but have a smaller range of frequencies around that frequency for which they resonate; the range of frequencies for which the oscillator resonates is called the bandwidth. Thus, a high $\mathrm{Q}$ tuned circuit in a radio receiver would be more difficult to tune, but would have more selectivity [3], [4]; it would do a better job of filtering out signals from other stations that lie nearby on the spectrum. High Q oscillators oscillate with a smaller range of frequencies and are more stable. The quality factor of oscillators varies substantially from system to system. Tuning forks have quality factors around $Q=1000$. The quality factor of atomic clocks and some high-Q lasers can reach as high as $10^{11}$ and higher [5].

Signal-to-noise ratio (often abbreviated SNR or S/N) is a measure used in science and engineering to quantify how much a signal has been corrupted by noise [5]. It is defined as the ratio of signal power to the noise power corrupting the signal. A ratio higher than 1:1 indicates more signal than noise. While SNR is commonly quoted for electrical signals, it can be applied to any form of signal (such as isotope levels in an ice core or biochemical signaling between cells) [4]. In less technical terms, signal-to-noise ratio compares the level of a desired signal (such as music) to the level of background noise. The higher the ratio, the less obtrusive the background noise is. "Signal-to-noise ratio" is sometimes used informally to refer to the ratio of useful information to false or irrelevant data in a conversation or exchange. For example, in online 
discussion forums and other online communities, off-topic posts and spam are regarded as "noise" that interferes with the "signal" of appropriate discussion. In a digitized measurement, the number of bits used to represent the measurement determines the maximum possible signal-to-noise ratio. This is because the minimum possible noise level is the error caused by the quantization of the signal (quantization noise) [6]. This noise level is non-linear and signal-dependent; different calculations exist for different signal models. Quantization noise is modeled as an analog error signal summed with the signal before quantization ("additive noise") [7].

This theoretical maximum SNR assumes a perfect input signal. If the input signal is noisy, the measurement noise may be larger than the quantization noise. Real analog-to-digital converters also have other sources of noise that further decrease the SNR compared to the theoretical maximum from the idealized quantization noise. Although noise levels in a digital system can be expressed using SNR, it is more common to use $\mathrm{E}_{\mathrm{b}} / \mathrm{N}_{\mathrm{o}}$ the energy per bit per noise power spectral density. The modulation error ratio (MER) is a measure of the SNR in a digitally modulated signal.

\section{NoISE SOURCES, Modulation AND CODING}

Noise is a significant issue in every communication system. In the optical world (especially in WDM) there are many sources of noise. The good news is that most of the noise sources are so small that may be ignored. In other cases the action can take to mitigate one form of noise also mitigates many others. The dominant noise sources in WDM systems are amplifier noise (ASE) and thermal noise in the receivers. However, in the design of any system it is very important to be aware of all the potential sources of noise so that they can be avoided or mitigated [8].

Modulation is the process of conveying a message signal, for example, a digital bit stream or an analog audio signal, inside another signal that can be physically transmitted. Modulation of a sine waveform is used to transform a base band message signal to a pass band signal, for example, a radio-frequency signal (RF signal). In radio communications, cable TV systems or the public switched telephone network for instance, electrical signals can only be transferred over a limited pass band frequency spectrum, with specific (non-zero) lower and upper cutoff frequencies [9]. In optical communication, there are two major modulation techniques: Electro-Absorption modulator and Mach-Zehnder modulator.

Electro-Absorption Modulator (EAM). Electro-Absorption modulator is a semiconductor device which can be used for modulating the intensity of a laser beam via an electric voltage. A change in the absorption spectrum caused by an applied electric field, which changes the band gap energy (thus the photon energy of an absorption edge) but usually, does not involve the excitation of carriers by the electric field. The EAM is candidate for use in external modulation links in telecommunications [10]. They can be operated at very high speed; a modulation bandwidth of tens of gigahertz can be achieved, which makes these devices useful for optical fiber communication. A convenient feature is that an EAM can be integrated with distributed feedback laser diode on a single chip to form a data transmitter in the form of a photonic integrated circuit. Compared with direct modulation of the laser diode, a higher bandwidth and reduced chirp can be obtained.

Mach-Zehnder Modulator. A Mach-Zehnder modulator is a intensity modulating signal light, using a simple drive circuit for the modulating voltage. The modulator includes two waveguides with respective multiple quantum well (MQW) structures. Well layers of the MQW structures of the two optical waveguides have different thicknesses or are made from different materials so the phase of light propagating through one waveguide advances and through the other waveguide is delayed in response to the same applied voltage. The phase-changed light signals are combined as an output light signal that is intensity modulated.

There are different types of coding are used such as NRZ, RZ, AMI, Manchester, Differential Manchester and Multi-state Coding. If the bit stream is to be sent as simply the presence or absence of light on the fiber (or as changes of voltage on a wire) then the simplest NRZ coding is possible. In this method a one bit is represented as the presence of light and a zero bit is represented as the absence of light [3]. This method of coding is used for some very slow speed optical links but has been replaced by other methods for most purposes. In RZ coding the signal returns to the zero state every bit time such as, a " 1 " bit is represented by a "ON" laser state for only half a bit time. In a restricted bandwidth environment (such as in most electronic communications) there are two different line states required to represent a bit (at least for a " 1 " bit) and this type of coding is not desired. AMI (Alternate Mark Inversion) is a synchronous clock encoding technique which uses bipolar pulses to represent logical 1 value. The alternating coding prevents the build-up of a D.C voltage level down the cable. This is considered an advantage since the cable may be used to carry a small D.C. current to power intermediate equipment such as line repeaters. Manchester encoding is a type of digital encoding that is used in data transmission. Within the structure for Manchester encoding, the data bits in the transmission are represented by a series of states that occur in a logical sequence. This approach to data transmission is somewhat different, as many encoding methods tend to assign a high or low state of voltage to each bit and use that information as the criteria for affecting the transfer of the bits. Differential Manchester encoding is a method of encoding data in which data and clock signals are combined to form a single self-synchronizing data stream. It is a differential encoding, using the presence or absence of transitions to indicate logical value. This gives it several advantages over standard Manchester encoding. Multi-state codes is a method in the electronic systems where both signal amplitude and phase are used to create unique line states representing particular bit combinations.

\section{ERROR DETECTION AND CORRECTION METHODOLOGY}

The general idea for achieving error detection and correction is to add some redundancy (i.e., some extra data) to a message, which receivers can use to check consistency of the delivered message and to recover data determined 
erroneous. Error-detection and correction schemes can be either systematic or non-systematic. In a systematic scheme, the transmitter sends the original data, and attaches a fixed number of check bits (or parity data), which are derived from the data bits by some deterministic algorithm. If only error detection is required, a receiver can simply apply the same algorithm to the received data bits and compare its output with the received check bits; if the values do not match, an error has occurred at some point during the transmission. In a system that uses a non-systematic code the original message is transformed into an encoded message that has at least as many bits as the original message. If the channel capacity cannot be determined or is highly varying, an error-detection scheme may be combined with a system for retransmissions of erroneous data. This is known as automatic repeat request (ARQ) and is most notably used in the Internet. An alternate approach for error control is hybrid automatic repeat request (HARQ) which is a combination of ARQ and error-correction coding [3].

Error detection is most commonly realized using a suitable hash function (or checksum algorithm). A hash function adds a fixed-length tag to a message, which enables receivers to verify the delivered message by recomputing the tag and comparing it with the one provided. There exists a vast variety of different hash function designs. However, some are of particularly widespread use because of either their simplicity or their suitability for detecting certain kinds of errors (e.g., the cyclic redundancy check's performance in detecting burst errors). Random error correcting codes based on minimum distance coding can provide a suitable alternative to hash functions when a strict guarantee on the minimum number of errors to be detected is desired.

\section{OPTICAL FIBER}

An optical fiber is a thin, flexible, transparent fiber that acts as a waveguide, or "light pipe", to transmit light between the two ends of the fiber. Optical fibers are widely used in fiber-optic communications, which permits transmission over longer distances and at higher bandwidths (data rates) than other forms of communication. There are two basic types of fiber: Single Mode Optical Fiber and Multi Mode Optical Fiber. Multiple color coded 900 um tight buffered fibers can be packed tightly together in a compact cable structure, an approach widely used indoors; these cables are called tight buffered cables [3]. Tight buffered cables are used to connect outside plant cables to terminal equipment, and also for linking various devices in a premises network. Multi-fiber, tight buffered cables often are used for intra-building, risers, general building and plenum applications. Tight-buffered cables are mostly built for indoor applications, although some tight buffered cables have been built for outdoor applications too. Elements in a tight buffered fiber optic cable: Multiple 900 um tight buffered fibers (stranded around the central strength member), Central strength member (in the center of the cable), Ripcord (for easy removal of outer jacket), Outer jacket (also called sheath, PVC is most common for indoor cables because of its flexible, fire-retardant and easy extrusion characteristics).

On the other hand multiple (up to 12) 250 um coated fibers (bare fibers) can be put inside a color coded, flexible plastic tube, which usually is filled with a gel compound that prevents moisture from seeping through the hollow tube. Buffer tubes are stranded around a dielectric or steel central member. Aramid Yarn is used as primary strength member. Then an outer polyethylene jacket is extruded over the core [9]. These cables are called loose tube cables. Loose tube structure isolates the fibers from the cable structure. This is a big advantage in handling thermal and other stresses encountered outdoors. Loose-tube cables typically are used for outside-plant installation in aerial, duct and direct-buried applications. Elements in a loose tube fiber optic cable: Multiple 250 um coated bare fibers (in loose tube), one or more loose tubes holding 250um bare fibers [10]. Loose tubes strand around the central strength member. Moisture-blocking gel is used in each loose tube for water blocking and protection of 250 um fibers. Central strength member (in the center of the cable and is stranded around by loose tubes). Aramid Yarn as strength member and Ripcord for easy removal of outer jacket. Outer jacket (Polyethylene is most common for outdoor cables because of its moisture resistant, abrasion resistant and stable over wide temperature range characteristics).

TABLE I: GENERATED OTDR'S REPORT INFORMATIONS

\begin{tabular}{cc}
\hline \hline \multicolumn{2}{c}{ General Information } \\
Filename: Qubee0042c2_1310.sor & Cable ID: 20133bd \\
Test date: 6/14/2010 & Fiber ID: oou13dd \\
Test time: 9.49 PM(GMT+06.00) & Job ID: A1 \\
Location A: ABB & Location B: CDC \\
Unit model: FTB-7300D-236B-E1 & Unit's s/n: 372208 \\
Results & \\
Span length: $7.2554 \mathrm{~km}$ & Average Space loss: $0.102 \mathrm{~dB}$ \\
Span loss: $3.067 \mathrm{~dB}$ & Maximum Space loss: $0.264 \mathrm{~dB}$ \\
Average loss: $0.426 \mathrm{~dB} / \mathrm{km}$ & Span ORL: $<18.76 \mathrm{~dB}$ \\
Test Parameters & \\
Wave length: $1310 \mathrm{~nm}(9 \mathrm{um})$ & Duration: $30 \mathrm{~s}$ \\
Range: $10,0000 \mathrm{~km}$ & High Resolution: Yes \\
Pulse: $10,000 \mathrm{~ns}$ & Resolution $0.319 \mathrm{~m}$ \\
IOR: 1.467700 & Test Settings \\
Backscatter: $-79.44 \mathrm{~dB}$ & Space loss threshold: $0.020 \mathrm{~dB}$ \\
Helix factor: $0.00 \%$ & Reflectance threshold: $-72.0 \mathrm{~dB}$ \\
\hline \hline
\end{tabular}

\section{Optical Signal Loss AND Bit ERror RAte ANALysis}

\section{A. Optical Signal Loss Analysis}

In optical fiber light is represented as signal and this signal carry individual bit. Bit error is totally dependable on signal loss. To find out the bit error correction in optical fiber the practical works is accomplished in Link-3 to observe the signal loss in fiber optics communication. Optical Time Domain Reflectometer (OTDR) device has been used in this regard and three individual distances $(1.01 \mathrm{~km}, 53.045 \mathrm{~km}$, and $98.61 \mathrm{~km})$ is considered finding out the error [3]. The example (based on a practical experience at Link-3 fiber network) states the signal loss particularly. The object of the fiber optic network is to calculate the attenuation - limited fiber length based on a power budget equation then to simulate and verify that is meets the performance objectives. The power budget equation states that the transmitted power minus the receiver sensitivity must be greater than or equal to the sum of the power losses plus the power margin as: 


$$
P_{T}-S_{R}=A L_{F}+L_{C}+L_{A}+M
$$

where, $P_{T^{-}}$is the transmitter, $S_{R^{-}}$is the receiver sensitivity, $A^{-}$is the fiber attenuation, $L_{F^{-}}$is the fiber length, $L_{C^{-}}$is the coupling loss, $L_{A^{-}}$is the additional know losses and $M^{-}$is the power margin.

\section{B. Fiber Signal Losses Calculation}

In the experiment, the fiber's length, attenuation and splice loss of a relatively short unknown optical fiber link are measured using the OTDR at 1310 operating wavelengths. An example of OTDR generated report is shown in Table I and the results are depicted in Fig. 1 and ODTR event table is shown in Table II. The general information of Table II is shown in Table III.

In the event Table II, Number (Num.), Location/Length (Loca./L), Reflection (Refl.), Attenuation (Atten.), Cumulative (Cumul.), Launch Level (LL), Non-Reflective Fault (NRF), Reflective Fault (RF), Positive Fault (PF) are shown.

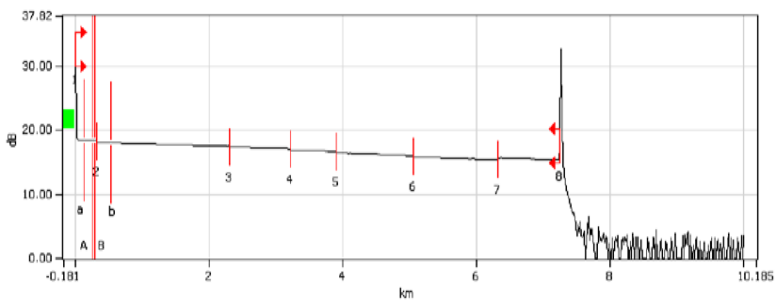

Fig. 1. OTDR generated test results.

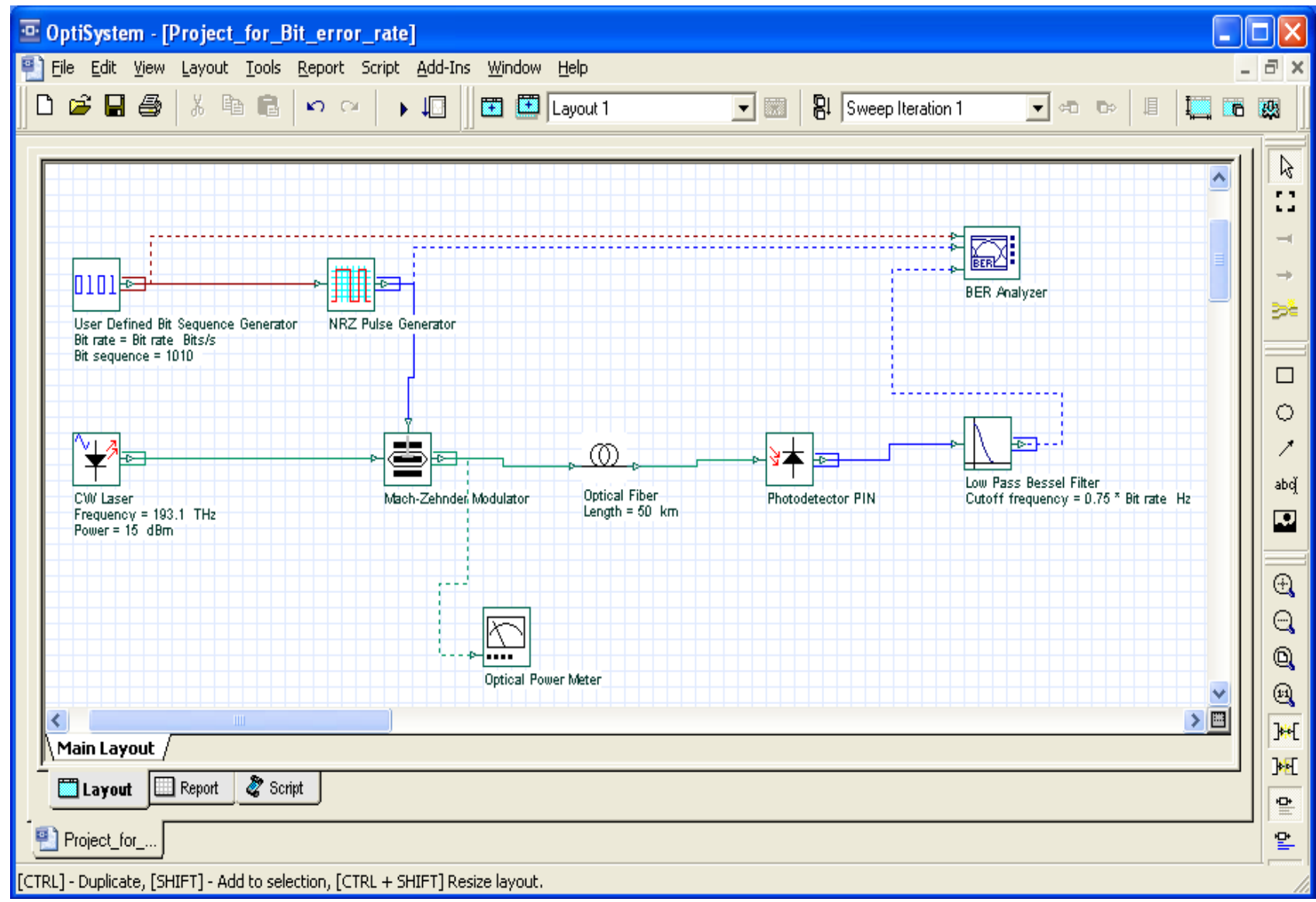

Fig. 2. The diagram of analyzed BER.

TABLE II: THE OTDR's EVENT TABLE

\begin{tabular}{lccccc}
\hline \hline Type Num & $\begin{array}{c}\text { Loca./L } \\
(\mathrm{km})\end{array}$ & $\begin{array}{c}\text { Loss } \\
(\mathrm{dB})\end{array}$ & $\begin{array}{c}\text { Refl. } \\
(\mathrm{dB})\end{array}$ & $\begin{array}{c}\text { Atten. } \\
(\mathrm{dB} / \mathrm{km})\end{array}$ & $\begin{array}{c}\text { Cumul. } \\
(\mathrm{dB})\end{array}$ \\
\hline LL 1 & 0.000 & & -23.2 & & 0.000 \\
Section & 0.321 & 0.080 & & 0.250 & 0.080 \\
NRF 2 & 0.321 & 0.284 & & & 0.364 \\
Section & 1.985 & 0.631 & & 0.318 & 0.995 \\
NRF 3 & 2.306 & 0.086 & & & 1.082 \\
$\quad$ Section & 0.904 & 0.280 & & 0.310 & 1.362 \\
NRF 4 & 3.210 & 0.158 & & & 1.520 \\
$\quad$ Section & 0.688 & 0.225 & & 0.326 & 1.744 \\
NRF 5 & 3.898 & 0.226 & & & 1.970 \\
$\quad$ Section & 1.157 & 0.497 & & 0.429 & 2.467 \\
NRF 6 & 5.055 & 0.172 & & & 2.639 \\
$\quad$ Section & 1.280 & 0.443 & & 0.346 & 3.082 \\
NRF 7 & 6.335 & -0.317 & -14.6 & & 2.765 \\
$\quad$ Section & 0.921 & 0.322 & & 0.350 & 3.087 \\
RF 8 & 7.255 & & & & 3.087 \\
\hline \hline
\end{tabular}

In Section II and V of the Table II, it is observed $0.28 \mathrm{~dB}$ and $0.22 \mathrm{~dB}$ signal loss, respectively. The signal loss point has to optimize the signal loss in fiber optic communication. The near-end zone of fiber A is fully visible before this reflection without any blinding by the front connector peak.

The marker and manual measurements information of the Table II are shown in Table III.

\section{Analysis of the BER}

The BER may be analyzed using stochastic computer simulations. If a simple transmission channel model and data source model is assumed, the BER may also be calculated analytically. In absence of available device for BER analysis, Optical Time Domain Reflectometer (OTDR) has been used for checking the signal loss in fiber optics and the OptiSystem 9.0 for analyzing the BER. The circuit diagram 
of Fig. 2 has been used to do this analysis.

TABLE III: THE OTDR's EVENT GENERAL INFORMATION

\begin{tabular}{cccclc}
\hline \hline \multicolumn{5}{c}{ Marker Information } \\
A: & $0.2588 \mathrm{~km}$ & $18.459 \mathrm{~dB}$ & $\mathrm{~B}: \quad 0.2895 \mathrm{~km}$ & $18.437 \mathrm{~dB}$ \\
A: & $0.1344 \mathrm{~km}$ & $18.461 \mathrm{~dB}$ & $\mathrm{~b}:$ & $0.5381 \mathrm{~km}$ & $18.038 \mathrm{~dB}$ \\
B-A: & $0.0306 \mathrm{~km}$ & $0.023 \mathrm{~dB}$ & & \\
\multicolumn{5}{c}{ Manual Measurements } \\
4-pt. ev. loss & $0.127 \mathrm{~dB}$ & A-B LSA att. & $-0.819 \mathrm{~dB} / \mathrm{km}$ \\
A-B LSA loss & $-0.025 \mathrm{~dB}$ & 3-pt. reflectance & $\ldots \ldots \ldots \ldots$ \\
2-pt. sect. att. & $0.733 \mathrm{db} / \mathrm{km}$ & A-B ORL & $54.70 \mathrm{~B}$ \\
\hline \hline
\end{tabular}

\section{The Components of the BER Diagram}

Input bit sequence, Signal pulse generator: RZ pulse generator and NRZ Pulse generator, Modulation Technique: Mach-Zehnder and Electro-Absorption, Optical input power: $20 \mathrm{~dB}$ and $15 \mathrm{~dB}$, cable $50 \mathrm{~km}$, A low pass filter, BER analyzer [6]. The following components of the BER diagram vary the result: Signal pulse generator, Modulation technique, Input optical Power. The four bits results are shown in Fig. 3 as well as in the Table IV whereas the eight bits results are shown in Fig. 4 as well as Table V.

TABLE V: EIGHT-BITS RESULT FROM BER ANALYSIS

\begin{tabular}{ccccc}
\hline \hline Input Bits & $\begin{array}{c}\text { Signal } \\
\text { Generator }\end{array}$ & $\begin{array}{c}\text { Modulation } \\
\text { Technique }\end{array}$ & $\begin{array}{c}\text { Optical } \\
\text { Power }(\mathrm{dB})\end{array}$ & $\begin{array}{c}\text { Min } \\
\text { BER }\end{array}$ \\
\hline 10101100 & RZ & MZ & 20 & $7.94587 \times \mathrm{e}^{-122}$ \\
10101100 & RZ & EAM & 20 & 0 \\
10101100 & NRZ & MZ & 20 & $3.09973 \times \mathrm{e}^{-28}$ \\
10101100 & NRZ & EAM & 20 & $3.84761 \times \mathrm{e}^{-28}$ \\
10101100 & RZ & MZ & 15 & $1.86539 \times \mathrm{e}^{-59}$ \\
10101100 & RZ & EAM & 15 & $7.99419 \times \mathrm{e}^{-132}$ \\
10101100 & NRZ & MZ & 15 & $1.06647 \times \mathrm{e}^{-37}$ \\
10101100 & NRZ & EAM & 15 & $4.50779 \times \mathrm{e}^{-53}$ \\
\hline \hline
\end{tabular}

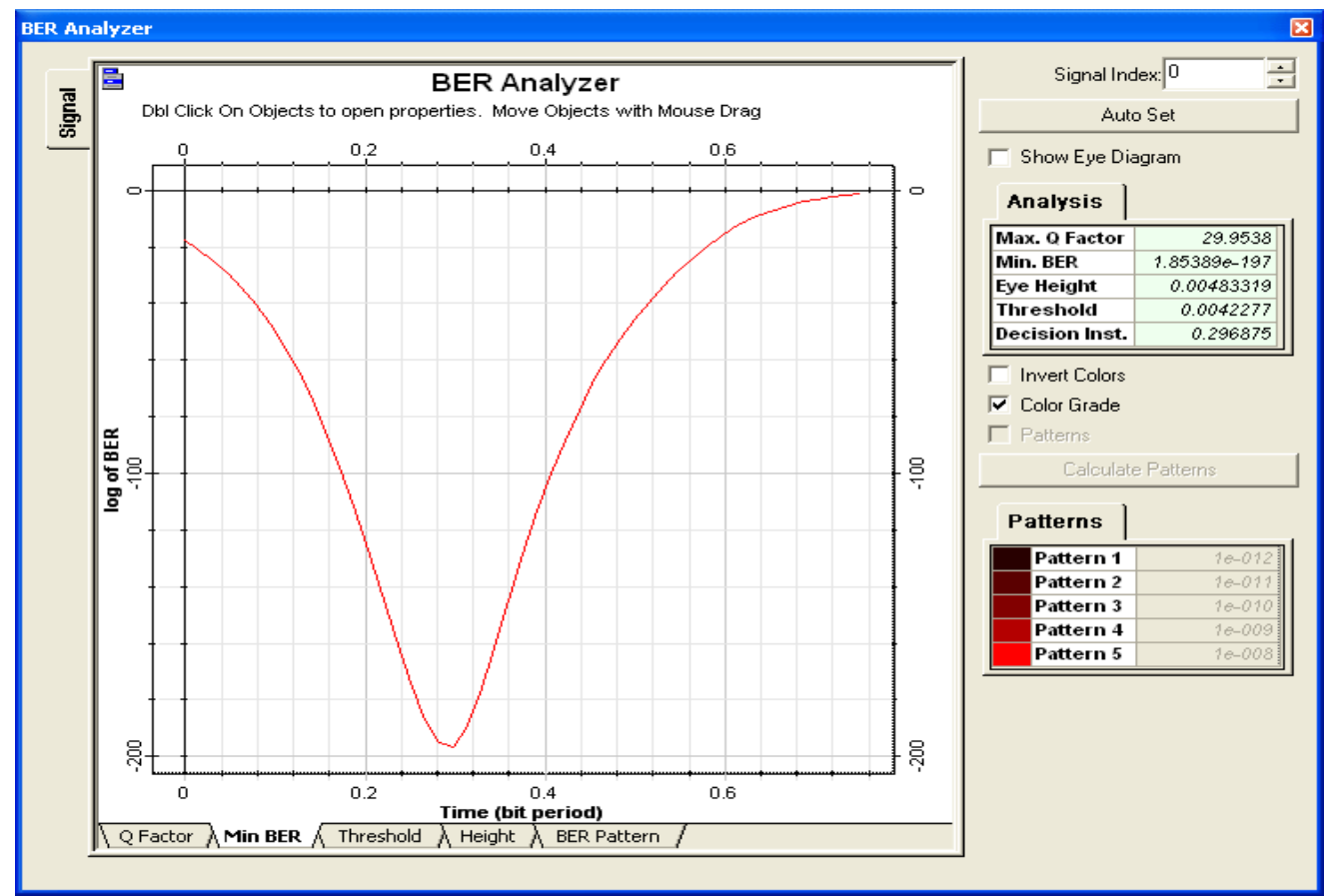

Fig. 3. Four bits BER analysis.

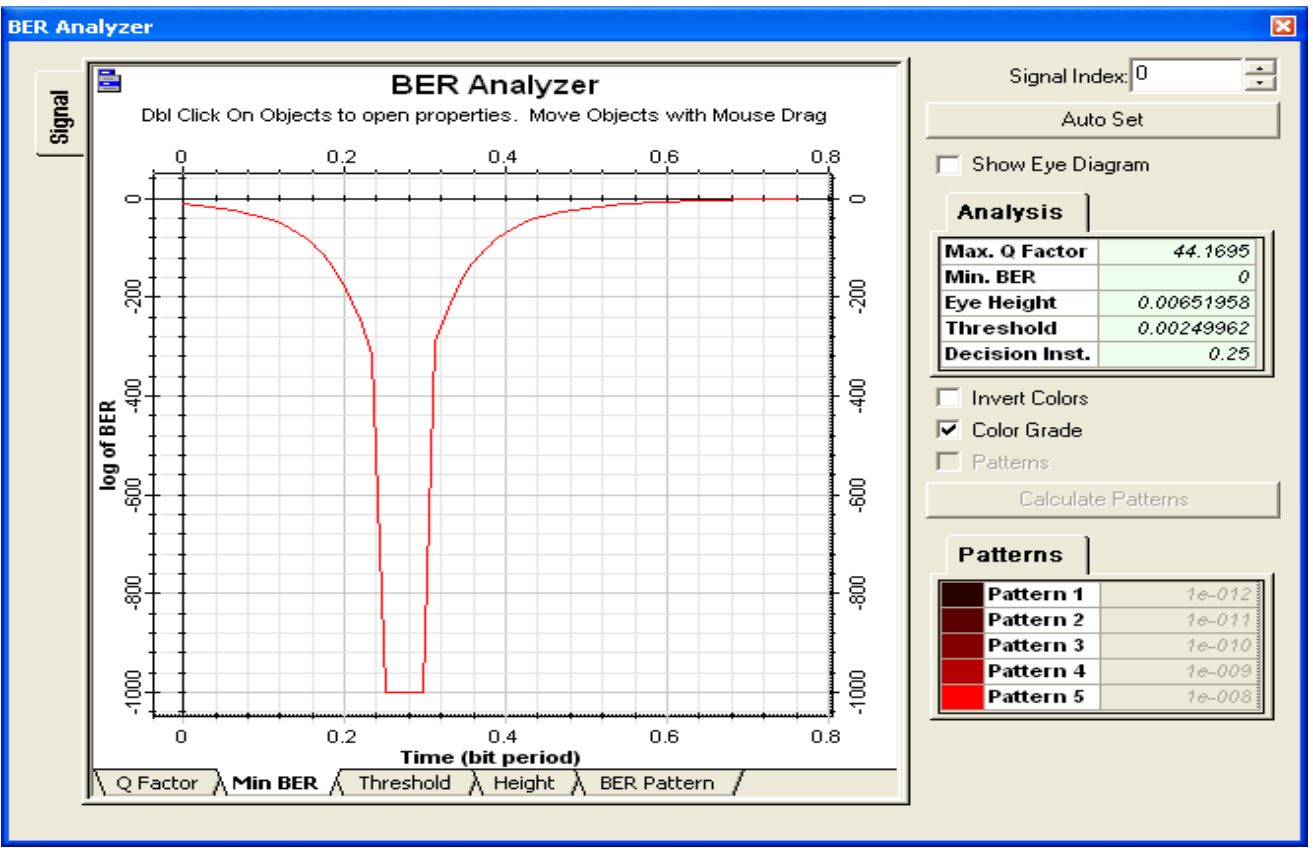

Fig. 4. Eight bits BER analysis. 
For four bits input 1010, Signal Generator: RZ, Modulation Technique: Mach Zehnder, Optical Power: 20 $\mathrm{dB}$, Minimum BER would be $1.85389 \times \mathrm{e}^{-197}$ which is shown in Fig. 3 as well as Table IV.

For the eight bits input 10101100, Signal Generator: RZ, Modulation Technique: Electro Absorption, Optical Power: $20 \mathrm{~dB}$, Min BER would be 0 as in Fig. 4 as well as Table V.

TABLE IV: FOUR BITS RESULT FROM BER ANALYSIS

\begin{tabular}{ccccc}
\hline $\begin{array}{c}\text { Input } \\
\text { Bits }\end{array}$ & $\begin{array}{c}\text { Signal } \\
\text { Generator }\end{array}$ & $\begin{array}{c}\text { Modulation } \\
\text { Technique }\end{array}$ & $\begin{array}{c}\text { Optical } \\
\text { Power }(\mathrm{dB})\end{array}$ & $\begin{array}{c}\text { Min. } \\
\text { BER }\end{array}$ \\
\hline 1010 & RZ & MZ & 20 & $1.85389 \mathrm{e}^{-197}$ \\
1010 & RZ & EAM & 20 & 0 \\
1010 & NRZ & MZ & 20 & 0 \\
1010 & NRZ & EAM & 20 & 0 \\
1010 & RZ & MZ & 15 & 0 \\
1010 & RZ & EAM & 15 & 0 \\
1010 & NRZ & MZ & 15 & $1.28085 \mathrm{e}^{-203}$ \\
1010 & NRZ & EAM & 15 & 0 \\
\hline \hline
\end{tabular}

\section{CONCLUSION}

In this study, a signal post processing approach has been suggested and tested on ODTR data signals that have been transmitted through single mode transmission system. The methods proposed to calculate the true average signal loss in the fiber optic communication; the single ended measurement offers a huge advantage in terms of time, logistics, result reliability and processing effort. The optimum solution reduces the bit error rate by using $\mathrm{RZ}$ signal generator through Electro Absorption modulation techniques. Numerical simulation shows a noticeable improvement of the system BER after optimization of the suggested processing operation on the detected electrical signals at central wavelengths in the region of $1310 \mathrm{~nm}$. The operation of optical transmission networks will be most important features in the near future to serve the ever increasing demand of IP networks. However, a lot of research works needs to be carried out to improve the increasing effective data transmission through these systems.

\section{ACKNOWLEDGMENT}

Warm expression and sincere thanks to Link3 Technologies Ltd., and system engineer Wahid Ferdous of Fiber optic department for his support to the experiment.

\section{REFERENCES}

[1] Wikipedia [Online]. http://en.wikipedia.org/wiki/Bit_error_rate

Available:

[2] G. Sha, A. Molina, and M. Blaze, "Keyboards and covert channels," in Proc. the 15th conference on USENIX Security Symposium, 2006, vol. 15 , p. 5.

[3] E. Sackinger, Broadband Circuits for Optical Fiber Communication, John Wiley and Sons, 2005, pp. 51.

[4] Signal-to-Noise Ratio. [Online] http://en.wikipedia.org/wiki/Signal-to-noise_ratio
[5] Techlib. [Online]. Available: http://www.techlib.com/reference/q.html

[6] I. B. Akca, A. Dana, A. Aydinli et al., "Electro-optic and electro-absorption characterization of InAs quantum dot waveguides," Optics Express, vol. 16, no. 5, pp. 3439-3444, 2008.

[7] Fixed-Point vs. Floating-Point DSP for Superior Audio, Rane Corporation technical library.

[8] X. Xu, B. Sun, P. R. Berman et al., "Coherent optical spectroscopy of a strongle driven quantum dot," Science, vol. 317, no. 5840, pp. 929-932, 2007.

[9] J. R. Barry, E. A. Lee, and D. G. Messerschmidt, Digital Communication, Kluwer Academic Publishers, 2004, pp. 203-205.

[10] S. G. Carter, "Quantum coherence in an optical modulator," Science, vol. 310, pp. 651, 2005.

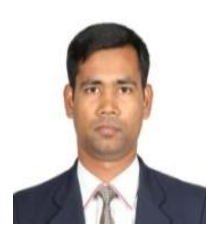

S. M. Jahangir Alam has been completed B.Sc. in computer science and engineering from Dhaka Intl. Univ., and he received his M.S degree in telecommunications from the Univ. of Info. Tech. \& Sci. (UITS), Dhaka, Bangladesh. He is a PhD candidate (June, 2014) in the Dept. of Mech. \& Electrical Engg. at Xiamen University, China.

He was a lecturer of electronic and communication engg. \& coordinator of the School of Comp. Sci. \& Engg. at UITS from 2006 to 2010. He is interested in vision \& image processing, TFT-LCD display defect detection, time series analysis, detection technology, and automation.

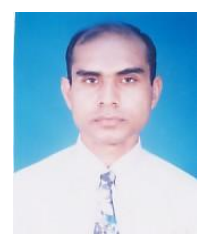

M. Rabiul Alam has been completed M.S. and awarded Ph.D. in electrical engineering from Moscow State Mining University, Moscow, Russia. From 2004 to 2006 he was a post-doctoral researcher (energy management) at the same University.

$\mathrm{He}$ is an associate professor and the head of the Department of Elect. \& Electronic Engg. at Hamdard University Bangladesh from 2012. During 2008-2012, he was an associate professor and the head of the Dept of Electrical and Electronic Engineering and dean of the School of Comp. Sci. and Engineering at Univ. of Info. Tech. and Scie. (UITS), Dhaka, Bangladesh. During 2007-2008 he was an associate professor of the Dept. of Electrical \& Electronic Engineering at International Univ. of Business Agriculture and Tech. (IUBAT), Dhaka, Bangladesh. His field of interest is power engineering, renewable energy and energy management.

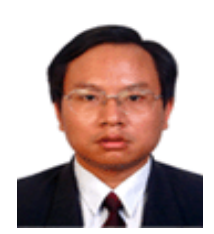

Guoqing Hu completed his B.S. and M.S. in the Dept. of Automation Control from Northwestern Polytechnical Univ. China and was awarded Ph.D. in mechanical engineering from Sichuan Univ. (PRC). In 1993-1995, he was a post-doctoral researcher in the Dept. of Mechanical Engineering, Shanghai Jiaotong University (PRC).

$\mathrm{He}$ is a professor of the Dept. of Mech. \& Elect. Engg. at Xiamen University, China since 1995. During 1993-1995, he was an associate professor in the Dept. of Mech. Engg., Shanghai Jiaotong Univ. In Jan 2000-Feb 2001, he was a visiting professor in the Dept. of Elect. Engg. \& Comp. Sci, Elect. Design Center, Case Western Reserve University (USA). From February 2002 to August 2002 he was a visiting professor in the Dept. of Automation \& CAE at The Chinese Univ. of Hong Kong (Hong Kong). His major research areas are advanced sensors, electromechanical control, mems, high temperature measure \& detection technology, industrial automation and robot, fluid transmission and control, etc.

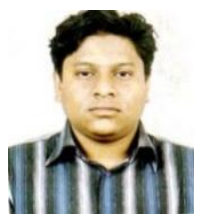

Md. Zakirul Mehrab has completed his BSc in Engg. from Dhaka Intl. Univ., Dhaka, Bangladesh and MS in telecommunications from UITS, Dhaka, Bangladesh.

$\mathrm{He}$ is an executive of Spark Technologies \& Consultancy Ltd., Bangladesh. His field of interest is data communications. 